\title{
Veille stratégique et prise de décision : une revue de la littérature
}

\section{Strategic Monitoring and Decision Making:}

A Literature Review

\section{Elsa Drevon, Dominique Maurel et Christine Dufour}

Volume 64, numéro 1, janvier-mars 2018

Survivre à la gestion?

URI : https://id.erudit.org/iderudit/1043720ar

DOI : https://doi.org/10.7202/1043720ar

Aller au sommaire du numéro

\section{Éditeur(s)}

Association pour l'avancement des sciences et des techniques de la documentation (ASTED)

\section{ISSN}

0315-2340 (imprimé)

2291-8949 (numérique)

Découvrir la revue

Citer cet article

Drevon, E., Maurel, D. \& Dufour, C. (2018). Veille stratégique et prise de décision : une revue de la littérature. Documentation et bibliothèques, 64(1), 28-34. https://doi.org/10.7202/1043720ar

\section{Résumé de l'article}

Le présent article vise à positionner la veille, et plus spécifiquement la veille stratégique, par rapport à la prise de décision, selon la littérature en sciences de gestion et en sciences de l'information. Trois principaux modèles de prise de décision seront présentés (première partie), à partir desquels ont pu être identifiés trois facteurs qui influencent la prise de décision (deuxième partie). Enfin, trois types de soutien qu'apporte la veille stratégique à la prise de décision seront mis en évidence (troisième partie), nous amenant à conclure que la veille stratégique contribue, théoriquement, à soutenir le processus de prise de décision en amont et en aval.
Tous droits réservés (c) Association pour l'avancement des sciences et des techniques de la documentation (ASTED), 2018
Ce document est protégé par la loi sur le droit d'auteur. L'utilisation des services d'Érudit (y compris la reproduction) est assujettie à sa politique d'utilisation que vous pouvez consulter en ligne.

https://apropos.erudit.org/fr/usagers/politique-dutilisation/ 


\section{VEILLE STRATÉGIQUE ET PRISE DE DÉCISION: UNE REVUE DE LA LITTÉRATURE}

\author{
Elsa DREVON \\ Candidate au doctorat en sciences \\ de l'information \\ École de bibliothéconomie et des sciences \\ de l'information, Université de Montréal \\ elsa.drevon@umontreal.ca
}

\author{
Dominique MAUREL \\ Professeure agrégée \\ École de bibliothéconomie et des sciences \\ de l'information, Université de Montréal \\ dominique.maurel@umontreal.ca
}

\author{
Christine DUFOUR \\ Professeure agrégée \\ École de bibliothéconomie et des sciences \\ de l'information, Université de Montréal \\ christine.dufour@umontreal.ca
}

Le présent article vise à positionner la veille, et plus spécifiquement la veille stratégique, par rapport à la prise de décision, selon la littérature en sciences de gestion et en sciences de l'information. Trois principaux modèles de prise de décision seront présentés (première partie), à partir desquels ont pu être identifiés trois facteurs qui influencent la prise de décision (deuxième partie). Enfin, trois types de soutien qu'apporte la veille stratégique à la prise de décision seront mis en évidence (troisième partie), nous amenant à conclure que la veille stratégique contribue, théoriquement, à soutenir le processus de prise de décision en amont et en aval.

\section{Strategic Monitoring and Decision Making:} A Literature Review

The purpose of this article is to situate intelligence, and more specifically strategic intelligence with regards to decision making as described in the management and information science literatures. The three principal models of decision making are presented (Part I), from which three factors influencing the decision making process were identified (Part II). Finally, three types of support provided by the strategic monitoring of decision making are highlighted (Part III), leading the authors to conclude that strategic monitoring theoretically contributes to the process prior to and following the actual decision.

\section{Introduction}

La veille stratégique est un processus systématique, continu, éthique et légal, de collecte, d'analyse, de traitement et de diffusion de l'information, celle-ci visant à aider les gestionnaires, la haute direction ou l'organisation dans son ensemble à prendre de meilleures décisions et à alimenter la réflexion stratégique, grâce à une meilleure compréhension de l'environnement externe et interne (Bergeron \& Hiller 2002 ; Guechtouli 2014 ; Drevon 2017). Dans la littérature scientifique et professionnelle, la veille stratégique s'inscrit généralement dans une perspective d'aide à la décision (Thiétart 1990; Audet 1998; Jakobiak 2001; Bergeron \& Hiller 2002; Brouard 2004; Guechtouli 2014), bien qu'elle vise aussi à soutenir la stratégie des organisations (Brouard 2004).

La prise de décision est un processus non linéaire qui consiste à effectuer un choix supposé éclairé par de l'information et motivé par l'expérience ou l'intuition du décideur, entre plusieurs options envisageables, dans le but d'agir dans un contexte social donné et en fonction des acteurs en jeu. Il ne s'agit pas nécessairement de chercher la meilleure solution, ni seulement de faire un simple choix parmi des options, mais de bien comprendre la situation pour décider de la solution la plus satisfaisante et la plus acceptable pour les parties prenantes (Simon 1977; March 1994; Zsambock \& Klein 2014). Dans plusieurs modèles qui seront exposés ultérieurement, l'information joue un rôle crucial, notamment dans le cas de décisions stratégiques (Citroën 2009).

Cependant, déjà à la fin des années 1970, alors que la veille ${ }^{1}$ commence à être implantée dans les grandes entreprises, deux chercheurs en management stratégique ${ }^{2}$ démontrent que l'information issue de la veille n'est pas utilisée par les décideurs. Selon Pfeffer \& Salancik (1978), d'une part, les décideurs ne sont pas familiers avec le vocabulaire employé par les veilleurs, et d'autre part, l'importance de l'information est jugée par ceux qui la collectent et non par les décideurs eux-mêmes, qui sont incapables de prédire de quelle

\footnotetext{
1. Dans notre article, la veille est un concept générique qui inclut différents types de veille, dont la surveillance de l'environnement (environmental scanning), la veille concurrentielle (competitive intelligence), la veille stratégique (strategic intelligence) et la veille informationnelle (current awareness).

2. Le management stratégique est une discipline au sein des sciences de gestion et qui a pour objet d'étude la stratégie dans les organisations.
} 
information ils auront besoin pour prendre une décision. "Those who provide information collect what they believe to be important. [...] A decision maker may be unable to predict what information he needs or would use." (Pfeffer \& Salancik 1978, 269) Plus récemment, Gilad \& Fuld (2016) ont mené une enquête auprès de 236 veilleurs et analystes stratégiques dans de grandes entreprises afin de mesurer l'utilisation de la veille. Selon cette étude, seule la moitié des entreprises utilisent l'information qu'elles collectent pour améliorer la prise de décision.

Par conséquent, il est nécessaire de revenir sur les études en sciences de l'information et en sciences de gestion qui ont étudié les soutiens qu'apporte la veille, et plus spécifiquement la veille stratégique, à la prise de décision dans les organisations, et ce, dans le but de mieux comprendre comment la veille stratégique contribuerait à soutenir la prise de décision. À cette fin, étant donné le peu de recherches menées sur le lien entre veille stratégique et prise de décision, nous avons mobilisé des écrits sur la veille en général, tout particulièrement ceux sur la surveillance de l'environnement (environmental scanning). Plusieurs auteurs ont en effet étudié le lien entre surveillance de l'environnement et prise de décision (Pfeffer \& Salancik 1978; Choo \& Auster 1994). Ces veilles, bien que différentes sur la portée des sources d'information exploitées ou les clientèles cibles, partagent en effet bien des similitudes quant aux caractéristiques du processus sous-jacent et aux objectifs d'aide à la décision (Drevon, Maurel \& Dufour 2016; Drevon 2017).

Le présent article constitue une partie de la revue de la littérature dans le cadre de notre thèse de doctorat portant sur la veille stratégique dans le secteur public de la santé. Il vise à positionner la veille, et plus spécifiquement la veille stratégique, par rapport à la prise de décision, selon la littérature en sciences de gestion et en sciences de l'information. Tout d'abord, cinq principaux modèles de prise de décision seront présentés en première partie. Ensuite trois facteurs qui influencent la prise de décision seront développés dans la deuxième partie. Enfin, dans la troisième partie, trois types de soutien qu'apporte la veille stratégique à la prise de décision seront mis en évidence.

\section{Qu'est-ce que la prise de décision?}

Trois principaux modèles provenant des sciences de gestion peuvent expliquer le processus de prise de décision, à savoir le modèle de la rationalité absolue, le modèle de la rationalité limitée et le modèle politique et de pouvoir. Ces modèles s'appliquent à tout type de décision, qu'elle soit opérationnelle, tactique ou stratégique, dans le contexte organisationnel public ou privé.

\section{Le modèle classique de la rationalité absolue}

Les économistes classiques, dont Adam Smith, ont développé le concept de rationalité absolue. Les individus cherchent à satisfaire leurs besoins au mieux: le consommateur maximise son utilité et le producteur son profit. L'homo oeconomicus ${ }^{3}$ dispose, en effet, d'une information complète sur la situation: il connaît toutes les solutions envisageables, ainsi que les conséquences de chacune de ces solutions. Il est donc en mesure de prendre la meilleure décision parmi les différentes solutions qui s'offrent à lui (Simon 1965).

Jugé aujourd'hui peu réaliste, ce modèle s'appliquerait éventuellement aux problèmes simples ou simplifiables (March 1994). La résolution du problème requiert de passer à travers plusieurs étapes:

1. Définir le problème;

2. Déterminer les solutions: dresser un inventaire de toutes les solutions possibles au problème;

3. Choisir la meilleure solution: procéder par élimination en posant le pour et le contre de chaque solution et en évaluant les conséquences de chaque solution;

4. Passer à l'action: résoudre le problème ;

5. Évaluer la décision.

Cependant, dès que le problème se complexifie comme c'est le cas dans le contexte organisationnel, le modèle de la rationalité absolue présente des limites. C'est pourquoi le prix Nobel d'économie Herbert Simon a proposé le modèle de la rationalité limitée, en 1977, en se basant sur la psychologie cognitive.

[I]l est nécessaire de revenir sur les études en sciences de l'information et en sciences de gestion qui ont étudié les soutiens qu'apporte la veille, et plus spécifiquement la veille stratégique, à la prise de décision dans les organisations [...].

\section{Le modèle de la rationalité limitée}

Le modèle de la rationalité limitée (Simon 1977) présuppose que l'incertitude est difficile à gérer et que l'information requise pour prendre des décisions est incomplète. En effet, le cerveau humain dispose de capacités cognitives limitées, c'est-à-dire qu'il est incapable d'absorber et d'analyser toute l'information disponible ni de réaliser l'ensemble des calculs nécessaires pour évaluer les conséquences de chaque solution

\footnotetext{
3. En économie néo-classique, l'homo oeconomicus est une représentation théorique du comportement de l'être humain.
} 
envisageable. La décision n'est donc pas la meilleure comme dans le modèle classique de la rationalité absolue, elle est celle que le décideur juge la plus satisfaisante: le décideur s'arrête au premier choix qu'il juge satisfaisant pour résoudre son problème en tenant compte des contraintes de son environnement (Simon 1977).

Dans les faits, la prise de décision se situerait dans un continuum entre rationalité absolue (dans le cadre de la résolution de problèmes simples par exemple) et rationalité limitée (dans le cadre de situations simples ou complexes) (Eisenhardt \& Zbarcaki 1992). Par exemple, lorsqu'un bibliothécaire décide d'acheter ou non une revue pour ses usagers, il prend sa décision en rassemblant de l'information sur l'offre, sur les préférences de ses usagers ou encore sur les statistiques de consultation de la revue dans le cas d'un renouvellement. Comme il ne dispose pas d'une information complète sur l'ensemble de ses usagers, par exemple, il choisit la solution qu'il juge la plus satisfaisante.

Plusieurs perspectives ont complété le modèle développé par Simon (1977). Ainsi, Mintzberg, Raisinghani \& Théorêt (1976) ont précisé que le processus de prise de décision ne suit pas nécessairement une logique séquentielle et que les décideurs passent beaucoup de temps à évaluer les différentes options. Eisenhardt (1989) a montré que les décideurs voient certes beaucoup d'informations, mais qu'ils ne se concentrent que sur quelques-unes d'entre elles, notamment dans le cadre des décisions stratégiques prises rapidement et en contexte incertain. Enfin, pour Fredrickson (1985), la prise de décision repose à la fois sur du rationnel et de l'intuitif.

D'ailleurs, l'intuition dans la prise de décision est aujourd'hui l'objet de plusieurs travaux, donc ceux de Zsambock \& Klein (2014). Selon leur modèle naturaliste, le décideur effectuerait un choix par expérience dans des situations complexes, incertaines, en temps réel et engageant de multiples acteurs. Il reconnaîtrait la situation par rapport à une expérience passée et mobiliserait cette expérience antérieure pour catégoriser rapidement les situations et pouvoir prendre ainsi une décision rapide satisfaisante.

Enfin, dans une forme extrême de la rationalité limitée, Cohen, March \& Olsen (1972) ont proposé le modèle poubelle, dans lequel la prise de décision serait la rencontre hasardeuse entre des solutions qui existent déjà dans l'organisation, des problèmes, des opportunités et des participants.

Le modèle de la rationalité limitée est encore aujourd'hui le plus utilisé dans la littérature scientifique pour expliquer le processus de prise de décision dans les organisations. Un autre modèle, né à la même époque, accorde, quant à lui, une place prépondérante aux conflits entre les multiples acteurs engagés dans une décision collective: le modèle politique et de pouvoir.

\section{Le modèle politique et de pouvoir}

Le modèle politique et de pouvoir (Cyert \& March 1963; Pettigrew 1973; Pfeffer \& Salancik 1974) puise ses racines dans la littérature en sciences politiques des années 1950 (Eisenhardt \& Zbaracki 1992). Une organisation serait avant tout un système politique (Pettigrew 1973) et la décision ne serait ni la maximisation du résultat, ni la satisfaction d'un décideur, mais la solution la plus acceptable pour tous les acteurs en jeu: la prise de décision reposerait sur un compromis entre les diverses parties prenantes qui disposent de pouvoirs et de sensibilités différentes et potentiellement conflictuelles (Cyert \& March 1963; Pettigrew 1973). D’ailleurs, les différents acteurs useraient de tactiques pour orienter la décision (Pfeffer \& Salancik 1974).

Dans le modèle politique et de pouvoir, la décision individuelle serait certes rationnelle, mais pas la décision collective: celle-ci serait le résultat des préférences et des intérêts des individus les plus puissants et influents (Pfeffer \& Salancik 1974). Par exemple, le choix d'un logiciel en bibliothèque peut être individuellement rationnel, mais la décision collective pourrait être avant tout le résultat des préférences des individus qui ont le plus de pouvoir dans l'organisation. Ce modèle reste aujourd'hui très important dans la littérature sur la prise de décision, bien que quelques auteurs aient souligné qu'il ne tient pas assez compte du fait que les individus vont toujours chercher ce qu'il y a de meilleur pour leur organisation (Eisenhardt \& Zbaracki 1992).

Ainsi, l'analyse des principaux modèles de prise de décision permet de dire qu'il n'y a pas qu'un seul modèle à retenir et que l'on peut tirer des enseignements de plusieurs d'entre eux. D'ailleurs, Eisenhardt \& Zbaracki $(1992,35)$ concluent que «strategic decision-making is best described by an interweaving of both boundedly rational and political processes». Aussi, la prise de décision est un processus non linéaire qui consiste à effectuer un choix supposé éclairé par de l'information et motivé par l'expérience ou l'intuition du décideur, entre plusieurs options envisageables, dans le but d'agir dans un contexte social donné et en fonction des acteurs en jeu. Il ne s'agit pas de chercher la meilleure solution, ni seulement de faire un simple choix parmi des options, mais de bien comprendre la situation pour décider de la solution la plus satisfaisante et la plus acceptable pour les parties prenantes.

À partir de ces principaux modèles, trois facteurs qui influencent la prise de décision peuvent être mis en évidence: (1) l'information, (2) l'expérience et l'intuition du décideur et (3) le contexte social.

\section{Quels facteurs influencent la prise de décision?}

L'information apparaît comme le principal facteur qui influence la prise de décision selon le modèle de la rationalité limitée. Toutefois, les récentes études sur l'influence de 
l'expérience et de l'intuition tendent à démontrer que la prise de décision reposerait en grande partie sur des éléments qui sembleraient irrationnels. Enfin, le contexte social joue un rôle majeur puisque le décideur serait avant tout un être social mû par un besoin de légitimité.

\section{L'information influence la prise de décision}

Pour les chercheurs en management stratégique Hambrick \& Mason (1984), l'analyse de la réalité par les décideurs passe par trois prismes: (1) un champ de vision limité par les capacités humaines cognitives et les valeurs des décideurs, qui ne peuvent pas analyser l'environnement dans sa totalité, (2) une perception sélective à l'intérieur de ce champ de vision limité et (3) une interprétation de la situation perçue. Les limites cognitives humaines et les valeurs des décideurs les amènent à percevoir et à interpréter une situation donnée. Par conséquent, les décideurs ne peuvent être certains de leur bonne compréhension de la situation et des solutions qui s'offrent à eux. L'information influencerait la prise de décision en permettant au décideur de mieux comprendre les contraintes dans son environnement, d'identifier des solutions, et d'envisager les conséquences de chacune d'entre elles. Par conséquent, l'information contribuerait à élargir le champ de vision limité des décideurs. Par exemple, un acheteur se renseignera au mieux sur les différents produits qui s'offrent à lui, et sera en mesure, sans pour autant connaître tous les produits existants, de décider rationnellement de celui qu'il juge le plus satisfaisant. Sa décision sera influencée par l'information dont il dispose sur les différents produits.

Plus précisément, selon Bergeron (2004), un décideur peut se trouver dans trois états différents au moment de prendre une décision : (1) un état de certitude, dans lequel l'environnement est déterminé et où l'information est complète quant aux diverses options possibles et à leurs conséquences; (2) un état de risque, dans lequel l'environnement est aléatoire, et le plus souvent, les options et les conséquences de ces options sont connues en fonction d'un calcul de probabilités qui

L'information influencerait la prise de décision en permettant au décideur de mieux comprendre les contraintes dans son environnement, d'identifier des solutions, et d'envisager les conséquences de chacune d'entre elles. Par conséquent, l'information contribuerait à élargir le champ de vision limité des décideurs. permet de modifier l'état de connaissance d'un individu (Choo 2002; Citroën 2009) contribuerait donc à améliorer sa prise de décision.

\section{L'expérience et l'intuition des décideurs influencent la prise de décision}

Les chercheurs du modèle naturaliste (Zsambock \& Klein 2014) insistent surtout sur l'expérience et l'intuition des décideurs. Selon eux, les décideurs ne réalisent pas des calculs de probabilités et n'utilisent pas non plus les arbres de décision quand ils prennent des décisions dans leur pratique de gestion. Même quand ils comparent des options, ils ne le font pas selon une évaluation systématique. Au contraire, ils cherchent à reconnaître le problème par rapport à une situation qu'ils ont déjà vécue. En mobilisant leur mémoire, les décideurs sont capables de prendre des décisions extrêmement rapidement (Zsambock \& Klein 2014). Par exemple, l'achat d'une revue en bibliothèque peut reposer sur l'expérience passée d'un bibliothécaire: par expérience, il sait que cette revue est utilisée par ses usagers. La décision ne repose donc pas sur une information donnée, mais bien sur l'expérience du décideur.

Selon Dane \& Pratt (2007), l'intuition est un processus inconscient de traitement de l'information, qui relie, de manière holistique, des éléments d'information épars, rapidement et immédiatement, et qui implique des jugements émotionnels. Le décideur émettrait donc une conclusion rapide en se basant sur des éléments d'information souvent disséminés et sans lien apparent, et en tenant compte de ses émotions. La prise de décision par intuition impliquerait l'acquisition implicite de connaissances issues de stimuli dans l'environnement (Dane \& Pratt 2007; Zsambock \& Klein 2014). Par exemple, encore une fois, l'achat d'une revue peut reposer sur l'intuition d'un bibliothécaire, qui considère que la revue en particulier pourrait rejoindre les usagers sans qu'il en ait pour autant la certitude. permet d'évaluer les risques associés au choix; (3) un état d'ignorance, dans lequel l'environnement est incertain et où il n'est pas possible d'effectuer un calcul de probabilités pour évaluer les conséquences de chaque solution. Lin, Cole \& Dalkir (2014) indiquent que l'information utile aux décideurs doit être évaluée selon sa pertinence, sa compréhensibilité, sa fiabilité, son accessibilité, et selon le temps et l'effort requis pour l'obtenir et l'exploiter. Si elle remplit les critères mentionnés précédemment, l'information permet de réduire l'incertitude, autrement dit de faire passer le décideur d'un état d'ignorance à un état de risque ou de certitude. Dans un contexte d'incertitude, l'information qui
Les décisions seraient non seulement influencées par l'information disponible et par l'expérience et l'intuition des décideurs, mais aussi par le contexte social.

\section{Le contexte social influence la prise de décision}

La théorie néo-institutionnelle (Di Maggio \& Powell 1983; Scott 2014) est connue pour son concept d'isomorphisme institutionnel: les organisations tendent à devenir similaires par recherche de légitimité. Par conséquent, les actions entreprises, dont la prise de décision, ne sont pas le fruit d'un calcul rationnel ou des forces concurrentielles sur 
le marché, mais le résultat de forces institutionnelles, telles que les normes et les préférences des décideurs, les politiques et la culture organisationnelles, les pressions gouvernementales et réglementaires, ou encore les normes au sein de l'industrie (Oliver 1997). La décision prise n'apparaît pas nécessairement la plus satisfaisante d'un point de vue économique, mais la plus acceptée socialement. Par exemple, le choix d'un logiciel en bibliothèque pourrait ne pas être économiquement le plus rentable, mais convenir aux traditions dans l'organisation, aux habitudes d'achat, ou encore aux préférences des décideurs.

Plus précisément, Oliver (1997) a comparé des décisions prises selon une rationalité économique (economic rationality) ou selon une rationalité normative (normative rationality). La rationalité économique est systématique, délibérée et orientée vers des buts économiques, tandis que la rationalité normative est habituelle, non rationnelle, et soumise aux normes et aux traditions dans l'organisation. La première est contrainte par les biais cognitifs des décideurs et l'incertitude de l'information comme dans la rationalité limitée, alors que la seconde l'est par le contexte historique et normatif de l'organisation. Par exemple, un bibliothécaire pourrait décider de renouveler l'abonnement à une revue, non pour des raisons économiquement rationnelles, mais pour limiter la résistance au changement, donc sous l'influence du contexte social.

Pour finir, dans la théorie néo-institutionnelle, les croyances et les pratiques sont tellement intériorisées par les acteurs qu'elles en deviennent invisibles. Par conséquent, selon Oliver (1997), certaines décisions organisationnelles apparaîtraient parfois irrationnelles d'un point de vue économique; elles seraient en réalité guidées par le contexte social.

La prise de décision organisationnelle est donc influencée par l'information, l'expérience et l'intuition du décideur, de même que par le contexte social. S'il apparaît dès lors évident que la veille stratégique soutient le processus de prise de décision en fournissant de l'information, comment y contribue-t-elle concrètement?

\section{Comment la veille stratégique contribue-t-elle à soutenir le processus de prise de décision?}

La veille stratégique permettrait de (1) réduire l'incertitude, (2) de détecter des signaux faibles et (3) de légitimer des décisions prises ou presque prises, et par conséquent de soutenir le processus de prise de décision en amont et en aval.

\section{La veille stratégique aiderait à réduire l'incertitude}

L'incertitude peut être causée par un manque ou une surabondance d'information (Daft 2007). Auster \& Choo (1994) sont les premiers à démontrer qu'il existe une forte corrélation entre le degré d'incertitude perçu par les gestionnaires et les activités de veille. Plus le degré d'incertitude est élevé, plus la fréquence des activités de veille augmente. Plus précisément, ces auteurs ont montré que l'information issue de la veille permet aux décideurs d'identifier des opportunités et des menaces, ce qui les amène à décider d'améliorer des projets (lancer un nouveau produit, formuler de nouvelles stratégies commerciales, etc.), et donc à prendre des décisions stratégiques relatives à ces projets. Ainsi, selon Bergeron et al. (2009), la veille stratégique aiderait à réduire l'incertitude en offrant une meilleure compréhension de l'environnement externe. Elle contribuerait donc à soutenir directement la prise de décision en amont. Par exemple, un bibliothécaire qui souhaite développer des services orientés usager pourrait mettre en place une veille stratégique des tendances et bonnes pratiques sur l'expérience utilisateur en bibliothèque. Cette veille stratégique l'amènerait à mieux comprendre le contexte de l'expérience utilisateur en bibliothèque, et ainsi contribuerait à réduire son incertitude dans le domaine avant de décider des services à développer.

Au-delà de la réduction de l'incertitude, la veille stratégique viserait surtout à donner du sens et donc à permettre une meilleure compréhension de l'environnement externe. Comme le soulignent Bergeron \& Hiller $(2002,368)$ : « [T]he purpose of $\mathrm{CI}^{4}$ (competitive intelligence) is to provide context and meaning to seemingly disparate facts, suppositions, and deductions surrounding a particular topic. » La veille stratégique ne consisterait donc pas uniquement à collecter de l'information, mais bien à donner du sens à des éléments d'informations disparates recueillis dans l'environnement pour les transformer en «connaissance d'action » (Bergeron et al. 2009, 192).

Enfin, il ne s'agit pas uniquement de rechercher de l'information pour répondre à une question, puisque le besoin d'information n'est pas connu à l'avance (Ayachi 2007), mais plutôt de provoquer des questionnements. Par exemple, la veille stratégique sur l'expérience utilisateur en bibliothèque pourrait répondre à un besoin informationnel encore vague, et susciterait plutôt des questionnements quant à la démarche elle-même. Le décideur modifierait son état de connaissance sur l'expérience utilisateur en bibliothèque et se rapprocherait d'un état plus certain, ce qui l'aiderait à décider par exemple de la première méthode à implanter pour concevoir des services orientés usager.

La veille stratégique aiderait donc à réduire l'incertitude, à donner du sens à des éléments d'informations disparates et à se questionner, et ce, en comprenant mieux l'environnement externe, ce qui soutiendrait directement la prise de décision en amont.

\footnotetext{
4. Bergeron \& Hiller (2002) emploient le terme competitive intelligence, mais leurs écrits en français, notamment Bergeron et al. (2009), utilisent le terme «veille stratégique.»
} 


\section{La veille stratégique aiderait à détecter des signaux faibles}

Grâce à la détection de signaux faibles, la veille stratégique soutiendrait la prise de décision fondée sur l'intuition en contribuant à acquérir des connaissances issues de stimuli de l'environnement externe et en nourrissant l'intuition et le bagage de connaissances des décideurs.

Les signaux faibles sont définis par Ansoff $(1975,22)$ comme: «[F]eatures of incipient changes that can help managers avoid surprises.» Selon Schoemaker, Day \& Snyder (2013), ils permettent de reconnaître dans l'environnement externe des microchangements qui se trouvent en périphérie de ce sur quoi les décideurs sont concentrés. Un des moyens de détecter des signaux faibles est la collecte d'information en provenance de sources d'information humaines (chercheurs, futuristes, etc.) et publiées (articles et ouvrages scientifiques, littérature grise, etc.) (El Akrouchi, Benbrahim \& Kassou 2015). Un signal faible peut également provenir de sources sensorielles (visuelles, auditives, olfactives, etc.). Théoriquement, la somme de l'ensemble des signaux faibles scrutés dans l'environnement externe grâce au processus de veille stratégique permettrait d'anticiper les tendances et les évolutions (Lesca \& Lesca 2011). Par exemple, un bibliothécaire pourrait décider d'implanter une méthode ethnographique qui a fait ses preuves pour concevoir des services orientés usager, en se basant sur son intuition qu'elle est la bonne pour sa bibliothèque. Les signaux faibles détectés dans sa veille sur le sujet auraient enrichi son bagage de connaissances sur plusieurs mois, et le bibliothécaire serait ainsi en mesure de prendre une décision rapide fondée sur son intuition.

Par la détection de signaux faibles, la veille stratégique permettrait donc de nourrir l'intuition et le bagage de connaissances des décideurs. Elle contribuerait à soutenir la prise de décision fondée sur l'intuition.

\section{La veille stratégique aiderait à légitimer des décisions déjà prises}

Dans certains cas, l'information fournie par la veille stratégique pourrait aussi servir à légitimer des décisions déjà prises ou presque prises.

Selon Feldman \& March (1981), l'information collectée, traitée et diffusée n'est pas utilisée de manière directe par les décideurs dans leur prise de décision. De plus, les décideurs collectent plus d'information qu'ils n'en utilisent, et demandent toujours de l'information complémentaire. Tout particulièrement dans les organisations bureaucratiques, la collecte d'information agit comme un rituel servant à démontrer ses compétences et son respect des normes comme doit le faire "un bon décideur» (Feldman \&
March 1981, 178, notre traduction). Plus que la prise de décision en elle-même, la collecte d'information soutiendrait donc surtout la recherche de légitimité du décideur, et de l'organisation dans son ensemble, notamment dans un contexte où les mesures de performance sont vagues et valorisent la rationalité des choix effectués (Feldman \& March 1981). Par exemple, dans le cadre de l'expérience utilisateur en bibliothèque, la décision d'implanter une méthode ethnographique en particulier pourrait reposer sur le fait qu'une autre bibliothèque l'a mise en place. Dans ce cas où la décision est sur le point d'être prise, la veille stratégique sur l'expérience utilisateur pourrait servir à la soutenir en fournissant une information qui justifie d'implanter cette méthode. Dans ce cas, la veille stratégique contribuerait donc à légitimer une décision déjà prise ou presque prise.

\section{Conclusion}

Les principaux modèles de la prise de décision, soit la rationalité absolue, la rationalité limitée et le modèle politique et de pouvoir, nous ont permis de mettre en évidence trois facteurs qui influencent la prise de décision dans le contexte des organisations: (1) l'information, (2) l'expérience et l'intuition des décideurs et (3) le contexte social. Le croisement de plusieurs écrits en sciences de gestion et en sciences de l'information permet d'avancer que la veille stratégique contribuerait, théoriquement, à soutenir la décision:

- En amont, grâce à une réduction de l'incertitude, une création de sens à partir d'éléments d'information épars, une meilleure compréhension de l'environnement, ainsi que la détection des signaux faibles qui nourrirait l'intuition des décideurs;

- En aval, grâce à la légitimité qu'apporte l'information issue de la veille stratégique dans certains cas de décisions déjà prises ou sur le point d'être prises.

Le soutien en amont de la décision servirait ainsi à conforter le décideur dans ses choix: sans pour autant l'amener dans un état de certitude totale sur les conséquences de ses choix, le décideur peut agir et décider de manière plus confortable. De même, le soutien en aval de la décision viserait à rassurer le décideur dans ses choix et à rassurer son organisation elle-même.

Mais, dans ce cas, est-ce que toute veille serait stratégique? Notre recherche doctorale portant sur la veille stratégique dans le secteur public de la santé contribuera à identifier plus précisément les usages et les non-usages de la veille, d'une part, dans le cadre de la prise de décision de gestionnaires, et d'autre part, dans le contexte du soutien à la stratégie des organisations. Elle permettra ainsi de mieux comprendre ce qu'est une veille réellement stratégique. 


\section{SOURCES CONSULTÉES}

Aguilar, Francis J. 1967. Scanning the business environment. New York: Macmillan.

Ansoff, H. Igor. 1975. Managing strategic surprise by response to weak signals. California Management Review 18 (2): 2-33.

Audet, Josée. 1998. La veille stratégique chez les PME québécoises, une étude de cas par comparaisons inter-sites. Thèse de doctorat. Québec: Université Laval.

Auster, Ethel \& Chun Wei Choo. 1994. CEO's, information and decisionmaking: scanning the environment for strategic advantage. Library Trends 43 (2): 206-225.

Ayachi, H'mida. 2007. L'adéquation entre le système d'information et la veille stratégique dans une activité de construction de sens. Management \& Avenir (12): 49-66.

Bergeron, Pierre G. 2004. La gestion moderne: une vision globale et intégrée ( 4 e éd.). Montréal: Gaëtan Morin éditeur.

Bergeron, Pierrette \& Christine A. Hiller. 2002. Competitive intelligence. Annual Review of Information Science and Technology 36 (1): 353-390.

Bergeron, Pierrette, Christine Dufour, Dominique Maurel \& Diane Mercier. 2009. La gestion stratégique de l'information. In Introduction aux sciences de l'information, sous la direction de JeanMichel Salaün et Clément Arsenault. Montréal: Les Presses de l'Université de Montréal, 183-205.

Brouard, François. 2004. Développement d'un outil diagnostique des pratiques existantes de la veille stratégique auprès des $P M E$. Thèse de doctorat. Trois-Rivières: Université du Québec à Trois-Rivières.

Choo, Chun Wei. 2002. Information management for the intelligent organization: The art of scanning the environment ( $3^{\mathrm{e}}$ éd.). Medford: Information Today.

Citroën, Charles Louis. 2009. Strategic decision-making processes: The role of information. Thèse de doctorat. Enschede: Université de Twente.

Cohen, Michael D., James G. March \& Johan P. Olsen. 1972. A garbage can model of organizational choice. Administrative Science Quarterly 17 (1): 1-25.

Cyert, Richard M. \& James G. March. 1963. A behavioral theory of the firm. Englewood Cliffs: Prentice-Hall.

Daft, Richard L. 2007. Organization theory and design (9e éd.). Mason: Thompson-South Western.

Dane, Erik \& Michael G. Pratt 2007. Exploring intuition and its role in managerial decision making. Academy of Management Review $32(1): 33-54$.

Di Maggio, Paul J. \& Walter W. Powell. 1983. The iron cage revisited: Institutional isomorphism and collective rationality in organizational fields. American Sociological Review 48: 147-160.

Drevon, Elsa, Dominique Maurel \& Christine Dufour. 2016. Veille stratégique et veille informationnelle dans un contexte d'organisation durable. In Actes du $7^{e}$ colloque Communication, Organisation, Société du Savoir et Information. Shippagan: Université de Moncton, 103-122.

Drevon, Elsa. 2017. La veille stratégique dans le secteur public de la santé au Québec: une étude de cas multiples. Proposition de recherche non publiée. Montréal: Université de Montréal.

Eisenhardt, Kathleen M. 1989. Making fast strategic decisions in high-velocity environments. Academy of Management Journal 32 (3): 543-576.

Eisenhardt, Kathleen M. \& Mark J. Zbaracki. 1992. Strategic decision making. Strategic Management Journal 13 (S2): 17-37.

El Akrouchi, Manal, Houda Benbrahim \& Ismail Kassou. 2015. Early warning signs detection in competitive intelligence. In Proceedings of the $25^{\text {th }}$ International Business Information Management Association Conference - Innovation Vision 2020. Amsterdam: IBIMA, 1014-1024.

Feldman, Martha S. \& James G. March. 1981. Information in organization as signal and symbol. Administrative Science Quarterly 26 (2): 171-186.

Fredrickson, James W. 1985. Effects of decision motive and organizational performance level on strategic decision processes. Academy of Management Journal 28: 821-843.

Gilad, Benjamin \& Leonard M. Fuld. 2016, 26 janvier. Only half of companies actually use the competitive intelligence they collect. Harvard business review. Consulté le: 6 novembre 2017. <hbr.org/ 2016/01/only-half-of-companies-actually-use-the-competitiveintelligence-they-collect>.

Guechtouli, Manel. 2014. Management des activités de veille stratégique: entre une organisation formelle et informelle. La Revue des Sciences de Gestion 2 (266): 23-31.

Hambrick, Donald C. \& Phyllis A. Mason. 1984. Upper echelons: The organization as a reflection of its top managers. The Academy of Management Review 9 (2): 193-206.

Jakobiak, François. 2001. Pratique de la veille technologique. Paris: Éditions d'Organisation.

Lesca, Humbert \& Nicolas Lesca. 2011. Weak signals for strategic intelligence: Anticipation tool for managers. Hoboken: John Wiley \& Sons.

Lin, Yang, Charles Cole \& Kimiz Dalkir. 2014. The relationship between perceived value and information source use during $\mathrm{km}$ strategic decision-making: A study of 17 Chinese business managers. Information Processing \& Management 50 (1): 156-174.

March, James G. 1994. A primer on decision-making: how decisions happen. New York: The Free Press.

Mintzberg, Henry, Duru Raisinghani \& André Théorêt. 1976. The structure of unstructured decision processes. Administrative Science Quarterly 21 (2): 246-275.

Oliver, Christine. 1997. Sustainable competitive advantage: Combining institutional and resource-based views. Strategic Management Journal 18 (9): 697-713.

Pettigrew, Andrew. 1973. Politics of organizational decision-making. Londres: Tavistock.

Pfeffer, Jeffrey \& Gerald R. Salancik. 1974. Organizational decision making as a political process: The case of a university budget. Administrative Science Quarterly 19: 135-151.

Pfeffer, Jeffrey \& Gerald R. Salancik. 1978. The external control of organizations. New York: Harper \& Row.

Schoemaker, Paul J. H., George S. Day \& Scott A. Snyder. 2013. Integrating organizational networks, weak signals, strategic radars and scenario planning. Technological Forecasting and Social Change 80 (4): 815-824.

Scott, W. Richard. 2014. Crafting an analytic framework. In Institutions and Organizations: Ideas, Interests, and Identities. Los Angeles: SAGE, 55-85.

Simon, Herbert A. 1965. The shape of automation: For men and management. New York: Harper \& Row.

Simon, Herbert A. 1977. The new science of management decision ( 2 éd.). Englewood Cliffs: Prentice-Hall.

Thiétart, Raymond Alain. 1990. La stratégie d'entreprise. Paris: Ediscience international.

Zsambock, Caroline E. \& Gary Klein. 2014. Naturalistic decision making. New York: Psychology Press. 